\title{
Os Subfibulare: A case report of painful fibular accessory ossicle
}

\author{
by Vasu Pai MS, D[Orth], National board [Orth], FICMR, FRACS, MCh[Orth] $]^{1}$
}

The Foot and Ankle Online Journal 3 (8): 3

A case report describing os subfibulare is presented. A 16 year-old female presents with a painful lump of the lateral malleolus. The os subfibulare formed a painful pseudo-arthrosis and the accessory ossicle was excised. Surgical excision resulted in complete, symptomatic relief. Case presentation includes radiographic and computed tomography findings with discussion of various accessory ossicles and their respective incidence in the lower extremity.

Key words: Os subfibulare, accessory ossicle, pseudo-arthrosis.

$\mathrm{O}$ s subfibulare is a rarely reported ossicle involving the inferior portion of the fibular tuberosity of the ankle. In general, accessory ossicles commonly observed in order of frequency of the lower extremity include: tibiale externum, os trigonum and os peroneum. Accessory bones that are rare in the foot include accessory interphalangeus, os peroneum, anamolous os calcaneum and talus, os trignum and os tibiale extenum. ${ }^{1-6}$ Os peroneum can fracture following an inversion and may mimic sport injury.

An accessory, distal focus of epiphyseal ossification may develop in either malleolus. These foci are not anatomically separate entities, even though they can appear to be radiographically. They usually are asymptomatic. However, they may be injured, either acutely or chronically.

Address correspondence to: Vasu Pai MS, D[orth], National board [Orth], FICMR, FRACS, MCh[Orth]. Gisborne Hospital, Ormond Road. Gisborne, NZ.

\footnotetext{
${ }^{1}$ Trainee house surgeon, Wellington Medical School, New Zealand.
}

If fractured, the injury can extend through a segment of the malleolus. An ossicle may also be avulsed as a ligament failure analogue, similar to a sleeve fracture of the patella. This is more common in the lateral than in the medial malleolus.

These avulsions, if not adequately diagnosed and treated, may progress to delayed union, nonunion, or a chronically painful ankle. We report a case of symptomatic os subfibulare.

\section{Case Report}

A 16 year-old female presented with pain of the right lateral ankle for six months duration. Pain was worse with shoe gear and painful when walking and running. She attempted various shoe gear and multiple courses of anti-inflammatory medication with no benefit or symptomatic relief. There was no evidence of instability or locking of the ankle joint. She denied any prior trauma. Clinical examination revealed a visible swelling $2 \mathrm{~cm}$ by $2 \mathrm{~cm}$ over the lateral malleolus. There was a palpable bony swelling that felt hard and fixed to the antero-inferior aspect of the lateral malleolus. It was tender on deep palpation. The ankle, subtalar and forefoot range of motion was within normal limits. 


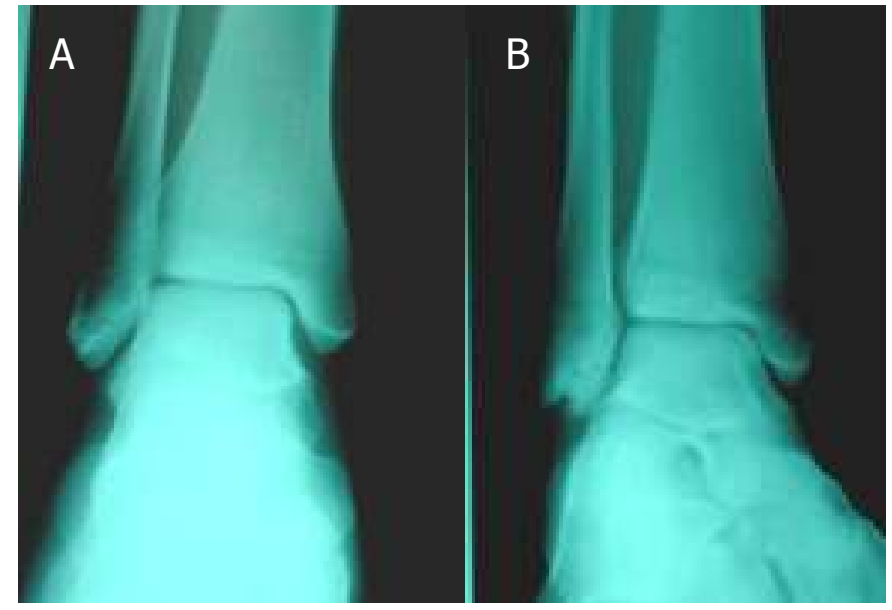

Figure 1 The anterior posterior (A) and Oblique (B) radiograph showing a large accessory ossicle or os subfibulare to the tip of the right lateral malleolus. The accessory ossicle is at the anterior medial portion of the malleolus giving it a bifid appearance.

Radiographic evaluation of the right ankle revealed an abnormality of the lateral malleolus. (Fig. 1A and B) There was an accessory ossicle at the lateral malleolus. The ossicle is enlarged and has a bifid appearance. Computed tomography (C'T) coronal and sagittal images show a single, anterior medial accessory ossicle of the fibula or os subfibulare. (Fig. 2) Threedimensional images show the ossicle with a local pseudo-arthrosis of the large os subfibulare along the distal anterior edge of the lateral malleolus. (Fig. 3)

Since symptoms were recalcitrant, exploration and removal of the ossicle was performed. An incision was centered over the area of edema and a pseudoarthrosis was demonstrated. The accessory ossicle was separated easily.

Part of the anterior talar fibular ligament was sutured to the lateral malleolus. Post-operatively, the ankle was placed in a posterior splint and held in neutral position for two weeks. After suture removal, the ankle was protected in range-of-motion brace for six weeks. One year post-operatively, the patient was noted to be totally asymptomatic.

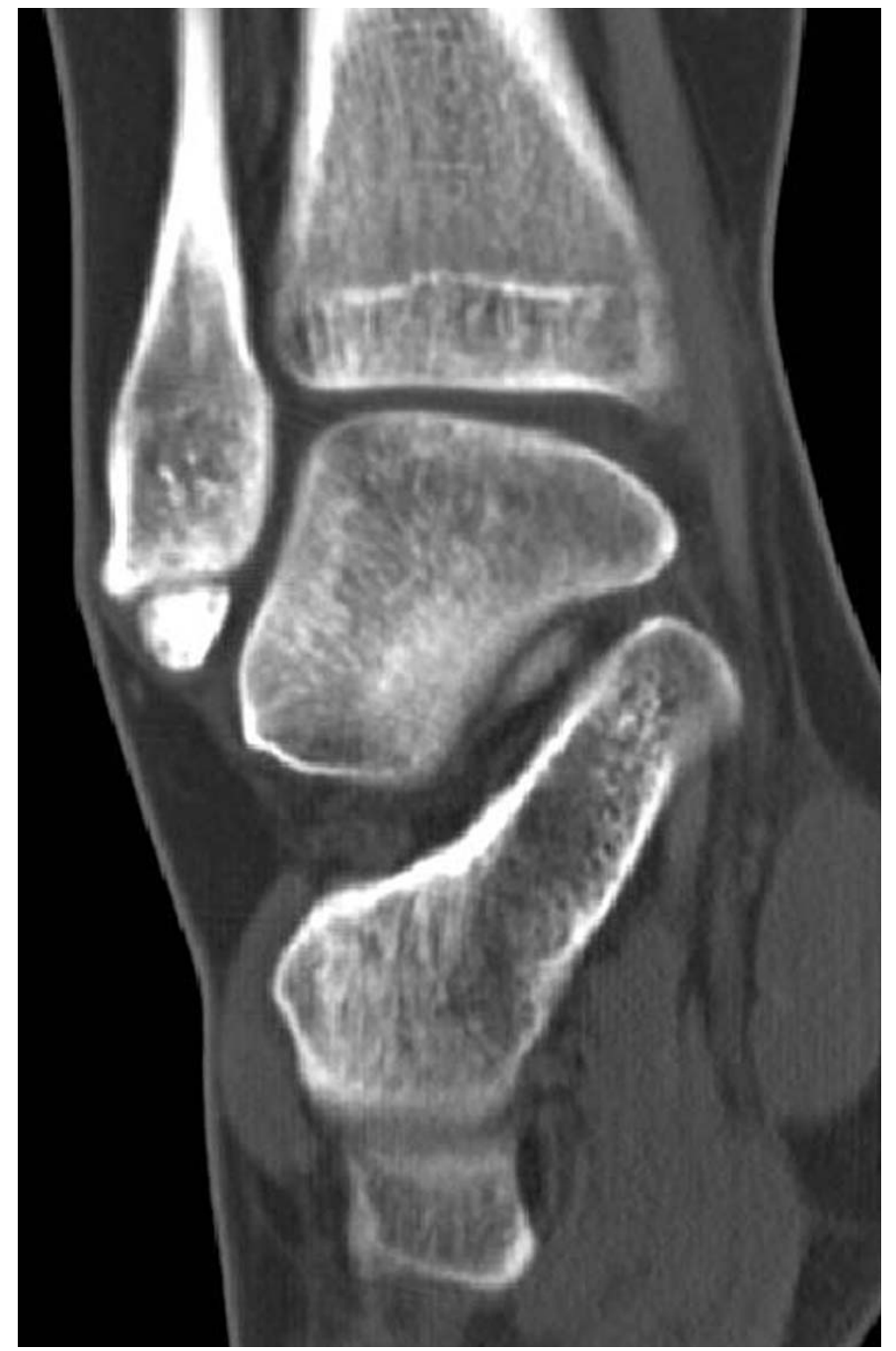

Figure 2 Computed tomography images show a fibular ossicle or os subfibulare at the distal end of the fibular with pseudo-arthrosis.

\section{Discussion}

Normally, the secondary center of ossification of the lateral malleolus appears during the first year of life, and fuses with the shaft at 15 years. Twenty two percent of normal children under the age of 16 have one or more accessory ossicles in the foot and ankle. ${ }^{8}$ The accessory ossicles most commonly observed, in order of frequency, are the tibiale externum, os trigonum and os peroneum.

In 3,460 radiographs of patients over 7 years of age, the os tibiale externum was the most common accessory bone. 


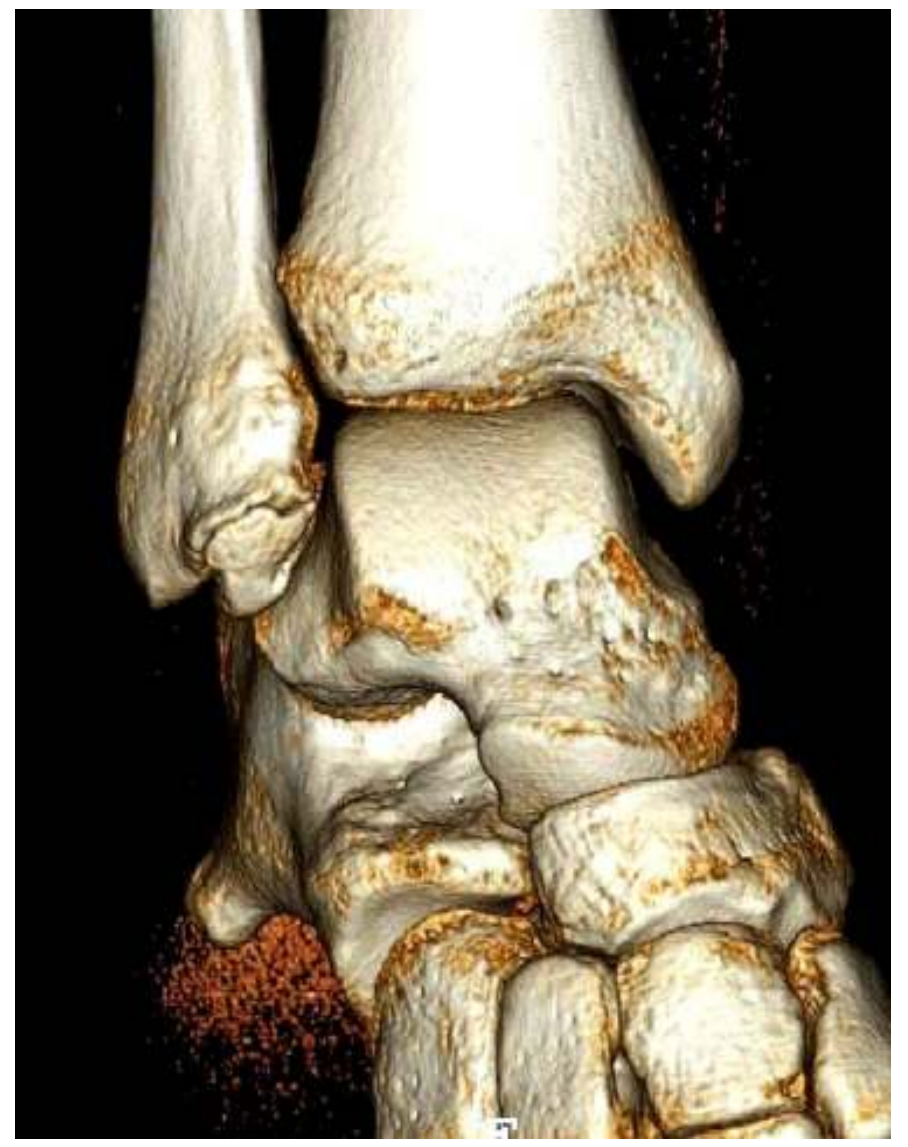

Figure 3 Three-dimensional computed tomography reveals a large accessory ossicle or os subfibulare to the tip of the lateral malleolus with pseudo-arthrosis of the fragment.

This is followed by os tibiale $(20 \%)$, os trigonum $(10 \%)$, os peroneum $(9 \%)$, os subfibulare $(2 \%)$, os supranaviculare $(1 \%)$ and os supratalare $(0.9 \%) .{ }^{9}$

The majority of os subfibulare are small. They are commonly separated from the tip of the lateral malleolus and are totally asymptomatic. Very rarely do they enlarge and become symptomatic. When symptomatic, it can be treated with anti-inflammatory drugs, physiotherapy and modified footwear. When symptoms are recalcitrant, surgical intervention is required. Griffith, et al., reported three children with symptomatic os subfibulare. All symptoms were relieved by excision of the ossicle and reconstitution of the collateral ligament. ${ }^{10}$
The precise cause of symptoms in patients is conjectural. The most likely explanation is that anomalous ossification centers, not yet fused to the body of the epiphysis, have been subjected to trauma, causing disruption to the fibrous or cartilaginous attachment and results in a fibrous union or pseudoarthrosis. Mechanical irritation or joint instability may produce local pain and tenderness and contribute to recurrent ankle sprains.

In this case, the operative findings revealed a mobile, separate ossicle attached to the lateral malleolus with an established pseudo-arthrosis. In summary, symptomatic os fibulare is extremely rare. When symptoms persist, surgical excision and repair of collateral ligament is indicated. ${ }^{11}$

\section{References}

1. Clarkson JH, Homfray T, Heron CW, Moss AL. CatelManzke syndrome: a case report of a female with severely malformed hands and feet. An extension of the phenotype or a new syndrome? Clin Dysmorphol Oct 2004 13(4):237-240. 2. Davies MB, Dalal S. Gross anatomy of the interphalangeal joint of the great toe: implications for excision of plantar capsular accessory ossicles. Clin Anat May 2005 18(4): 239-244. 3. Mellado JM, Ramos A, Salvado E, Camins A, Danus M, Sauri A. Accessory ossicles and sesamoid bones of the ankle and foot: imaging findings, clinical significance and differential diagnosis.Eur Radiol Dec 2003 Suppl. 6: L164-177.

4. Mosel LD, Kat E, Voyvodic F. Imaging of the symptomatic type II accessory navicular bone. Australas Radiol June 2004 48(2): 267-271.

5. Miller TT. Painful accessory bones of the foot. Semin Musculoskelet Radiol. Review June 2002 6(2):153-161. 6.Ogden JA. Anomalous multifocal ossification of the os calcis. Clin Orthop Relat Res. Jan-Feb 1982 (162): 112-118.

7. Saxena A. Unusual foot pathologies mimicking common sports injuries.J Foot Ankle Surg Jan-Feb 1993 32(1): 53-59. 8. Shands AR Jr. Accessory bones of foot: x-ray study of feet of 1,054 patients. South Med Surg 1931 93: 326-334.

9. Tsuruta T, Shiokawa Y, Kato A, Matsumoto T, Yamazoe Y, Oike T, Sugiyama T, Saito M. [Radiological study of the accessory skeletal elements in the foot and ankle (author's transl)]Nippon Seikeigeka Gakkai Zasshi April 1981 55(4): $357-$ 370 .

10.Griffith JD, Menelaus MB. Symptomatic ossicles of the lateral malleolus in children. JBJS March 1987 69B (2):317-9.

11..Mancuso JE, Hutchison PW, Abramow SP, Landsman MJ. Accessory ossicle of the lateral malleolus. J Foot Surg Jan-Feb 1991 30(1): 52-55. 